\title{
Existence of positive solutions for a system of Caputo fractional difference equations depending on parameters
}

\author{
Shugui Kang ${ }^{*}$, Huiqin Chen and Jianmin Guo
}

\section{"Correspondence:}

dtkangshugui@126.com

School of Mathematics and

Computer Sciences, Shanxi Datong

University, Datong, Shanxi 037009,

P.R. China

\section{Springer}

\begin{abstract}
We consider the existence of at least two positive solutions for a system of Caputo fractional difference equations $\Delta_{\mathrm{C}}^{v_{j}} y_{j}(t)=-\lambda_{j} f_{j}\left(y_{1}\left(t+v_{1}-1\right), \ldots, y_{n}\left(t+v_{n}-1\right)\right)$, subject to boundary conditions $y_{j}\left(v_{j}-3\right)=\Delta y_{j}\left(v_{j}+b\right)=\Delta^{2} y_{j}\left(v_{j}-3\right)=0$, where $2<v_{j} \leq 3$, $j=1, \ldots, n$. We use the Krasnosel'skil fixed point theorem to obtain the sufficient conditions of the existence of two positive solutions for this boundary value problem of Caputo fractional difference equations depending on parameters.
\end{abstract}

MSC: 26A33; 39A05; 39A12

Keywords: Caputo fractional difference; boundary value problem; fixed point theory; positive solution

\section{Introduction}

In this paper we consider a system of Caputo fractional difference boundary value problem (FBVP) of the form:

$$
\begin{aligned}
& \Delta_{\mathrm{C}}^{v_{j}} y_{j}(t)=-\lambda_{j} f_{j}\left(y_{1}\left(t+v_{1}-1\right), \ldots, y_{n}\left(t+v_{n}-1\right)\right), \\
& y_{j}\left(v_{j}-3\right)=\Delta y_{j}\left(v_{j}+b\right)=\Delta^{2} y_{j}\left(v_{j}-3\right)=0,
\end{aligned}
$$

where $t \in[0, b+1]_{\mathbb{N}_{0}}:=\{0,1, \ldots, b+1\}, b>3, \lambda_{j}>0,2<v_{j} \leq 3, f_{j}:[0,+\infty) \times \cdots \times[0,+\infty) \rightarrow$ $[0,+\infty)$ are continuous functions for each $j(j=1,2, \ldots, n) . \Delta_{C}^{v} y(t)$ is the standard Caputo difference.

Fractional difference equations have been of great interest recently. It is caused by intensive development of the theory of discrete fractional calculus itself, see [1-19] and the references therein. Abdeljawad [1] defined left and right Caputo fractional sums and differences, studied some of their properties. Holm [2] introduced the fractional sum and difference operators. He developed and presented a complete and precise theory for composing fractional sums and differences. Atici and Sengül [3] provided some analysis of discrete fractional variational problems, their paper also provided some initial attempts at using the discrete fractional calculus to model biological processes. Abdeljawad and Baleanu [4] defined the right fractional sum and difference operators and obtained many of their properties. Then by using those properties they obtained a by-part formula analogous to that in the usual fractional calculus. In [5] the authors studied the stability of

(c) 2015 Kang et al.; licensee Springer. This article is distributed under the terms of the Creative Commons Attribution 4.0 International License (http://creativecommons.org/licenses/by/4.0/), which permits unrestricted use, distribution, and reproduction in any medium, provided you give appropriate credit to the original author(s) and the source, provide a link to the Creative Commons license, and indicate if changes were made. 
discrete nonautonomous systems within the frame of the Caputo fractional difference by using the Lyapunov direct method. They discussed the conditions for uniform stability, uniform asymptotic stability, and uniform global stability. Mohammadi and Rezapour [6] discussed the existence and uniqueness of solutions for some nonlinear fractional differential equations via some boundary value problems by using fixed point results on ordered complete gauge spaces. Recently, Wu and Baleanu introduced some applications of the Caputo fractional difference to discrete chaotic maps in $[7,8]$.

In particular, the authors [9-19] developed some of the basic theory of fractional difference both IVPs and BVPs with delta derivative on the time scale $\mathbb{Z}$. In [10], we obtained some results on the existence of one or more positive solutions for the Caputo fractional boundary value problems by means of cone theoretic fixed point theorems. Thus, the fractional difference equation has recently attracted increasing attention from a growing number of researchers. However, systems of discrete fractional boundary value problems are limited (see [14-19]). Among them, Atici and Eloe [14] studied a linear system of fractional nabla difference equation with constant coefficients of the form

$$
\nabla_{0}^{v} y(t)=A y(t)+f(t), \quad t=1,2, \ldots
$$

where $0<v<1, A$ is an $n \times n$ matrix with constant entries, and $f$ are $n$-vector valued functions. The operator $\nabla_{0}^{v}$ is a Riemann-Liouville fractional difference. They constructed the fundamental matrix for the homogeneous system and the causal Green's function for the nonhomogeneous system.

In [15], the authors investigated the existence of solutions for a $k$-dimensional system of fractional finite difference equations:

$$
\begin{aligned}
& \Delta^{v_{1}} y_{1}(t)+f_{1}\left(y_{1}\left(t+v_{1}-1\right), y_{2}\left(t+v_{2}-1\right), \ldots, y_{k}\left(t+v_{k}-1\right)\right)=0, \\
& \Delta^{v_{2}} y_{2}(t)+f_{2}\left(y_{1}\left(t+v_{1}-1\right), y_{2}\left(t+v_{2}-1\right), \ldots, y_{k}\left(t+v_{k}-1\right)\right)=0, \\
& \ldots, \\
& \Delta^{v_{k}} y_{k}(t)+f_{k}\left(y_{1}\left(t+v_{1}-1\right), y_{2}\left(t+v_{2}-1\right), \ldots, y_{k}\left(t+v_{k}-1\right)\right)=0, \\
& y_{1}\left(v_{1}-2\right)=\Delta y_{1}\left(v_{1}+b\right)=0, \\
& y_{2}\left(v_{2}-2\right)=\Delta y_{2}\left(v_{2}+b\right)=0, \\
& \ldots, \\
& y_{k}\left(v_{k}-2\right)=\Delta y_{k}\left(v_{k}+b\right)=0,
\end{aligned}
$$

where $b \in \mathbb{N}_{0}, 1<v_{i} \leq 2, f_{i}: \mathbb{R}^{k} \rightarrow \mathbb{R}$ are continuous functions for $k=1,2, \ldots$. They investigated the existence of solutions for this $k$-dimensional system of fractional finite difference equations by using the Krasnosel'skii fixed point theorem.

In [16], Goodrich studied the following pair of discrete fractional boundary value problems:

$$
\begin{aligned}
& -\Delta^{v_{1}} y_{1}(t)=\lambda_{1} a_{1}\left(t+v_{1}-1\right) f_{1}\left(y_{1}\left(t+v_{1}-1\right), y_{2}\left(t+v_{n}-1\right)\right), \\
& -\Delta^{v_{2}} y_{2}(t)=\lambda_{2} a_{2}\left(t+v_{1}-1\right) f_{2}\left(y_{1}\left(t+v_{1}-1\right), y_{2}\left(t+v_{n}-1\right)\right),
\end{aligned}
$$




$$
\begin{array}{ll}
y_{1}\left(v_{1}-2\right)=\psi_{1}\left(y_{1}\right), & y_{2}\left(v_{2}-2\right)=\psi_{2}\left(y_{2}\right), \\
y_{1}\left(v_{1}+b\right)=\phi_{1}\left(y_{1}\right), & y_{2}\left(v_{2}+b\right)=\phi_{2}\left(y_{2}\right),
\end{array}
$$

where $t \in[0, b]_{\mathbb{N}_{0}}:=\{0,1, \ldots, b\}, \lambda_{1}, \lambda_{2}>0, v_{1}, v_{2} \in(1,2]$. Goodrich obtained the existence of at least one positive solution to this problem by means of the Krasnosel'skii theorem for cons.

In [18], the authors considered the existence of at least one positive solution to the discrete fractional system:

$$
\left\{\begin{array}{l}
-\Delta^{v_{1}} y_{1}(t)=\lambda_{1} f_{1}\left(t+v_{1}-1, y_{1}\left(t+v_{1}-1\right), y_{2}\left(t+v_{2}-1\right)\right), \quad t \in[1, b+1], \\
-\Delta^{v_{2}} y_{2}(t)=\lambda_{2} f_{2}\left(t+v_{2}-1, y_{1}\left(t+v_{1}-1\right), y_{2}\left(t+v_{2}-1\right)\right), \quad t \in[1, b+1], \\
y_{1}\left(v_{1}-2\right)=y_{1}\left(v_{1}+b+1\right)=0, \\
y_{2}\left(v_{2}-2\right)=y_{2}\left(v_{2}+b+1\right)=0,
\end{array}\right.
$$

where $v_{1}, v_{2} \in(1,2]$.

Following this trend, in [19], we discussed the boundary value problems of fractional difference system of the form

$$
\begin{aligned}
& -\Delta^{v_{j}} y_{j}(t)=\lambda_{j} f_{j}\left(y_{1}\left(t+v_{1}-1\right), \ldots, y_{n}\left(t+v_{n}-1\right)\right), \\
& y_{j}\left(v_{j}-2\right)=\psi_{j}\left(y_{j}\right), \quad y_{j}\left(v_{j}+b\right)=\phi_{j}\left(y_{j}\right),
\end{aligned}
$$

where $t \in[0, b]_{\mathbb{N}_{0}}:=\{0,1, \ldots, b\}, \lambda_{j}>0,1<v_{j} \leq 2, f_{j}:[0,+\infty) \times \cdots \times[0,+\infty) \rightarrow[0,+\infty)$ are continuous functions. For each $j$ we have that $\psi_{j}, \phi_{j}: \mathbb{R}^{b+3} \rightarrow \mathbb{R}(j=1,2, \ldots, n)$ are given functions. We obtained the sufficient conditions for the existence of two positive solutions to the boundary value problem of a fractional difference system. In this paper we open our studies in this field. We establish some conditions on parameters $\lambda_{i}$ which are able to guarantee that FBVP (1.1)-(1.2) has at least two positive solutions and one positive solution, respectively, based on the Krasnosel'skii theorem.

This paper is organized as follows. In Section 2, we provide basic definitions and demonstrate some lemmas in order to prove our main results. In Section 3, we establish some results for the existence of at least two positive solutions to FBVP (1.1)-(1.2), and we conclude with an example explicating our main result.

\section{Preliminaries}

In this section, we present some basic definitions in the discrete fractional calculus and establish some lemmas.

Definition 2.1 [1] We define

$$
t^{(v)}=\frac{\Gamma(t+1)}{\Gamma(t+1-v)}
$$

for any $t$ and $v$ for which the right-hand side is defined. We also appeal to the convention that if $t+1-v$ is a pole of the gamma function and $t+1$ is not a pole, then $t^{(v)}=0$.

Definition 2.2 [1] The $v$ th fractional sum of a function $f$ is defined by

$$
\Delta^{-v} f(t)=\frac{1}{\Gamma(\nu)} \sum_{s=a}^{t-v}(t-s-1)^{\frac{v-1}{}} f(s)
$$


for $v>0$ and $t \in\{a+v, a+v+1, \ldots\}=\mathbb{N}_{a+v}$. We also define the $v$ th Caputo fractional difference for $v>0$ by

$$
\Delta_{C}^{v} f(t)=\Delta^{-(n-v)} \Delta^{n} f(t)=\frac{1}{\Gamma(n-v)} \sum_{s=a}^{t-(n-v)}(t-s-1)^{\frac{n-v-1}{2}} \Delta^{n} f(s),
$$

where $n-1<v \leq n$.

Lemma 2.3 $[1,2]$ Assume that $v>0$ and $f$ is defined on domains $\mathbb{N}_{a}$, then

$$
\Delta_{a+(n-v)}^{-v} \Delta_{C}^{v} f(t)=f(t)-\sum_{k=0}^{n-1} c_{k}(t-a)^{\underline{k}},
$$

where $c_{i} \in \mathbb{R}, i=0,1, \ldots, n-1 ; n-1<v \leq n$.

Lemma 2.4 [2] Let $f: \mathbb{N}_{a+v} \times \mathbb{N}_{a} \rightarrow \mathbb{R}$ be given. Then

$$
\Delta\left(\sum_{s=a}^{t-v} f(t, s)\right)=\sum_{s=a}^{t-v} \Delta_{t} f(t, s)+f(t+1, t+1-v) \quad \text { for } t \in \mathbb{N}_{a+v} .
$$

In order to get our main results, we now state an important lemma. This lemma gives a representation for the solution of (1.1)-(1.2), provided that the solution exists.

Lemma $2.5[10]$ Let $2<v \leq 3$ and $g:[v-2, v-1, \ldots, v+b]_{\mathbb{N}_{v-2}} \rightarrow \mathbb{R}$ be given. Then the solution of the FBVP

$$
\begin{aligned}
& \Delta_{C}^{v} y(t)=-g(t+v-1), \\
& y(v-3)=\Delta y(v+b)=\Delta^{2} y(v-3)=0
\end{aligned}
$$

is given by

$$
y(t)=\sum_{s=0}^{b+1} G(t, s) g(s+v-1)
$$

where the Green's function $G:[v-2, v-1, \ldots, v+b]_{\mathbb{N}_{v-2}} \times[0, b+1]_{\mathbb{N}_{0}} \rightarrow \mathbb{R}$ is defined by

$$
G(t, s)=\frac{1}{\Gamma(v)} \begin{cases}(v-1)(t-v+3)(v+b-s-1)^{\frac{v-2}{2}} & \\ -(t-s-1) \frac{v-1}{2}, & 0 \leq s<t-v+1 \leq b+1 \\ (v-1)(t-v+3)(v+b-s-1)^{\frac{v-2}{2},} & 0 \leq t-v+1 \leq s \leq b+1 .\end{cases}
$$

Remark Notice that $G(v-3, s)=0, G(t, b+2)=0 . G$ could be extended to $[v-3, v+$ $b]_{\mathbb{N}_{v-3}} \times[0, b+2]_{\mathbb{N}_{0}}$, so we only discuss on $(t, s) \in[v-2, v+b]_{\mathbb{N}_{v-2}} \times[0, b+1]_{\mathbb{N}_{0}}$.

Lemma 2.6 [10] The Green's function $G$ satisfies the following conditions:

(i) $G(t, s)>0,(t, s) \in[v-2, v+b]_{\mathbb{N}_{v-2}} \times[0, b+1]_{\mathbb{N}_{0}}$.

(ii) $\max _{t \in[v-2, v+b]_{\mathbb{N}_{v-2}}} G(t, s)=G(v+b, s), s \in[0, b+1]_{\mathbb{N}_{0}}$.

(iii) $\min _{\frac{v+b}{4} \leq t \leq \frac{3(v+b)}{4}} G(t, s) \geq \frac{1}{4} \max _{t \in[v-2, v+b]_{\mathbb{N}_{v-2}}} G(t, s)=\frac{1}{4} G(v+b, s), s \in[0, b+1]_{\mathbb{N}_{0}}$. 
The proofs of Lemma 2.5 and Lemma 2.6 can be found in [10], so we omit their proofs. Let

$$
\mathcal{B}_{j}:=\left\{y_{j}:\left[v_{j}-3, v_{j}+b\right]_{\mathbb{N}_{v_{j}-3}} \rightarrow \mathbb{R}, y_{j}\left(v_{j}-3\right)=\Delta y_{j}\left(v_{j}+b\right)=\Delta^{2} y_{j}\left(v_{j}-3\right)=0\right\}
$$

be equipped with the usual maximum norm $\|\cdot\|$, it is easy to verify that $\mathcal{B}_{j}$ is the Banach space. Then we put $\mathcal{K}:=\mathcal{B}_{1} \times \mathcal{B}_{2} \times \cdots \times \mathcal{B}_{n}$. By equipping $\mathcal{K}$ with the norm

$$
\left\|\left(y_{1}, \ldots, y_{n}\right)\right\|=\left\|y_{1}\right\|+\cdots+\left\|y_{n}\right\|,
$$

it follows that $(\mathcal{K},\|\cdot\|)$ is a Banach space.

Now consider the operator $T: \mathcal{K} \rightarrow \mathcal{K}$ defined by

$$
T\left(y_{1}, \ldots, y_{n}\right)\left(t_{1}, \ldots, t_{n}\right)=\left(T_{1}\left(y_{1}, \ldots, y_{n}\right)\left(t_{1}\right), \ldots, T_{n}\left(y_{1}, \ldots, y_{n}\right)\left(t_{n}\right)\right)
$$

where we define $T_{j}: \mathcal{K} \rightarrow \mathcal{B}_{j}$ by

$$
T_{j}\left(y_{1}, \ldots, y_{n}\right)\left(t_{j}\right)=\lambda_{j} \sum_{s=0}^{b+1} G_{j}\left(t_{j}, s\right) f_{j}\left(y_{1}\left(s+v_{1}-1\right), \ldots, y_{n}\left(s+v_{n}-1\right)\right) .
$$

Let $I:=\left[\frac{v_{1}+b}{4}, \frac{3\left(v_{1}+b\right)}{4}\right] \times \cdots \times\left[\frac{v_{n}+b}{4}, \frac{3\left(v_{n}+b\right)}{4}\right]$. In the sequel, we shall also make use of the cone

$$
\begin{aligned}
\Lambda:= & \left\{\left(y_{1}, \ldots, y_{n}\right) \in \mathcal{K}: y_{1}, \ldots, y_{n} \geq 0,\right. \\
& \left.\min _{\left(t_{1}, \ldots, t_{n}\right) \in I}\left[y_{1}\left(t_{1}\right)+\cdots+y_{n}\left(t_{n}\right)\right] \geq \frac{1}{4}\left\|\left(y_{1}, \ldots, y_{n}\right)\right\|\right\} .
\end{aligned}
$$

Lemma 2.7 Let $T$ be the operator defined as in (2.3). Then $T: \Lambda \rightarrow \Lambda$.

Proof We show first that for $\left(y_{1}, \ldots, y_{n}\right) \in \mathcal{K}$, by the definitions $T_{j}(j=1,2, \ldots, n)$, it is clear that

$$
T_{j}\left(y_{1}, \ldots, y_{n}\right)\left(t_{j}\right) \geq 0, \quad j=1,2, \ldots, n .
$$

On the other hand, we show that

$$
\min _{\left(t_{1}, \ldots, t_{n}\right) \in I}\left[T_{1}\left(y_{1}, \ldots, y_{n}\right)\left(t_{1}\right)+\cdots+T_{n}\left(y_{1}, \ldots, y_{n}\right)\left(t_{n}\right)\right] \geq \frac{1}{4}\left\|T\left(y_{1}, \ldots, y_{n}\right)\right\|
$$

for $\left(y_{1}, \ldots, y_{n}\right) \in \mathcal{K}$. In fact, by Lemma 2.6(iii), we have

$$
\begin{aligned}
& \min _{t_{j} \in\left[\frac{v_{j}+b}{4}, \frac{3\left(v_{j}+b\right)}{4}\right]} T_{j}\left(y_{1}, \ldots, y_{n}\right)\left(t_{j}\right) \\
& \geq \min _{t_{j} \in\left[\frac{b+v_{j}}{4}, \frac{3\left(v_{j}+b\right)}{4}\right]} \lambda_{j} \sum_{s=0}^{b+1} G_{j}\left(t_{j}, s\right) f_{j}\left(y_{1}\left(s+v_{1}-1\right), \ldots, y_{n}\left(s+v_{n}-1\right)\right)
\end{aligned}
$$




$$
\begin{aligned}
& \geq \lambda_{j} \sum_{s=0}^{b+1} \frac{1}{4} G_{j}\left(v_{j}+b, s\right) f_{j}\left(y_{1}\left(s+v_{1}-1\right), \ldots, y_{n}\left(s+v_{n}-1\right)\right) \\
& \geq \frac{1}{4} \max _{t_{j} \in\left[v_{j}-2, v_{j}+b\right]_{v_{v_{j}-2}}} \lambda_{j} \sum_{s=0}^{b+1} G_{j}\left(t_{j}, s\right) f_{j}\left(y_{1}\left(s+v_{1}-1\right), \ldots, y\left(s+v_{n}-1\right)\right) \\
& =\frac{1}{4}\left\|T_{j}\left(y_{1}, \ldots, y_{n}\right)\right\|
\end{aligned}
$$

for $\left(y_{1}, \ldots, y_{n}\right) \in \mathcal{K}$ and $j=1,2, \ldots, n$. Then we obtain

$$
\begin{aligned}
& \min _{\left(t_{1}, \ldots, t_{n}\right) \in I}\left[T_{1}\left(y_{1}, \ldots, y_{n}\right)\left(t_{1}\right)+\cdots+T_{n}\left(y_{1}, \ldots, y_{n}\right)\left(t_{n}\right)\right] \\
& \geq \min _{\left(t_{1}, \ldots, t_{n}\right) \in I} T_{1}\left(y_{1}, \ldots, y_{n}\right)\left(t_{1}\right)+\cdots+\min _{\left(t_{1}, \ldots, t_{n}\right) \in I} T_{n}\left(y_{1}, \ldots, y_{n}\right)\left(t_{n}\right) \\
& \geq \frac{1}{4}\left\|T_{1}\left(y_{1}, \ldots, y_{n}\right)\right\|+\cdots+\frac{1}{4}\left\|T_{n}\left(y_{1}, \ldots, y_{n}\right)\right\| \\
& \geq \frac{1}{4}\left\{\left\|T_{1}\left(y_{1}, \ldots, y_{n}\right)\right\|+\cdots+\left\|T_{n}\left(y_{1}, \ldots, y_{n}\right)\right\|\right\} \\
& =\frac{1}{4}\left\|T\left(y_{1}, \ldots, y_{n}\right)\right\|
\end{aligned}
$$

for $\left(y_{1}, \ldots, y_{n}\right) \in \mathcal{K}$. So, we conclude that $T: \Lambda \rightarrow \Lambda$. This completes the proof.

Theorem 2.8 Let $f_{j}:[0,+\infty) \times \cdots \times[0,+\infty) \rightarrow[0,+\infty)$ be given for $j=1, \ldots, n$. If $\left(y_{1}, \ldots, y_{n}\right) \in \mathcal{K}$ is a fixed point of $T$, then $\left(y_{1}, \ldots, y_{n}\right) \in \mathcal{K}$ is a solution of FBVP (1.1)-(1.2).

Proof Suppose that the operator $T$ has a fixed point, say $\left(y_{1}, \ldots, y_{n}\right) \in \mathcal{K}$. Let $\left(t_{1}, \ldots, t_{n}\right) \in$ $\mathbb{N}_{v_{1}-2} \times \cdots \times \mathbb{N}_{v_{n}-2}$, then we have

$$
y_{j}\left(t_{j}\right)=T_{j}\left(y_{1}, \ldots, y_{n}\right)\left(t_{j}\right), \quad j=1,2, \ldots, n,
$$

where $T_{j}$ is defined as in (2.4). It is easy to check that

$$
T_{j}\left(y_{1}, \ldots, y_{n}\right)\left(v_{j}-3\right)=0
$$

and

$$
\begin{aligned}
\Delta & T_{j}\left(y_{1}, \ldots, y_{n}\right)\left(v_{j}+b\right) \\
= & T_{j}\left(y_{1}, \ldots, y_{n}\right)\left(v_{j}+b+1\right)-T_{j}\left(y_{1}, \ldots, y_{n}\right)\left(v_{j}+b\right) \\
= & \lambda_{j} \sum_{s=0}^{b+1} G_{j}\left(v_{j}+b+1, s\right) f_{j}\left(y_{1}\left(s+v_{1}-1\right), \ldots, y_{n}\left(s+v_{n}-1\right)\right) \\
& \quad-\lambda_{j} \sum_{s=0}^{b+1} G_{j}\left(v_{j}+b, s\right) f_{j}\left(y_{1}\left(s+v_{1}-1\right), \ldots, y_{n}\left(s+v_{n}-1\right)\right) \\
= & \lambda_{j} \sum_{s=0}^{b+1}\left[G_{j}\left(v_{j}+b+1, s\right)-G_{j}\left(v_{j}+b, s\right)\right] f_{j}\left(y_{1}\left(s+v_{1}-1\right), \ldots, y_{n}\left(s+v_{n}-1\right)\right) \\
= & \frac{\lambda_{j}}{\Gamma(v)} \sum_{s=0}^{b+1}\left[\left(v_{j}-1\right)\left(v_{j}+b+1-v_{j}+3\right)\left(v_{j}+b-s-1\right) \frac{v_{j}-2}{-}-\left(v_{j}+b+1-s-1\right) \underline{v_{j}-1}\right.
\end{aligned}
$$




$$
\begin{aligned}
& \left.-\left(v_{j}-1\right)\left(v_{j}+b-v_{j}+3\right)\left(v_{j}+b-s-1\right) \frac{v_{j}-2}{-}+\left(v_{j}+b-s-1\right) \frac{v_{j}-1}{}\right] \\
& \times f_{j}\left(y_{1}\left(s+v_{1}-1\right), \ldots, y_{n}\left(s+v_{n}-1\right)\right) \\
= & \frac{\lambda_{j}}{\Gamma(v)} \sum_{s=0}^{b+1}\left\{\left(v_{j}-1\right)\left(v_{j}+b-s-1\right) \frac{v_{j}-2}{}\right. \\
& \left.-\left[\frac{\left(v_{j}+b-s\right) \Gamma\left(v_{j}+b-s\right)}{(b-s+1) \Gamma(b-s+1)}-\frac{\Gamma\left(v_{j}+b-s\right)}{\Gamma(b-s+1)}\right]\right\} \\
& \times f_{j}\left(y_{1}\left(s+v_{1}-1\right), \ldots, y_{n}\left(s+v_{n}-1\right)\right) \\
= & \frac{\lambda_{j}}{\Gamma(v)} \sum_{s=0}^{b+1}\left[\left(v_{j}-1\right)\left(v_{j}+b-s-1\right) \frac{v_{j}-2}{(b-s+1) \Gamma(b-s+1)}-\frac{\left(v_{j}-1\right) \Gamma\left(v_{j}+b-s\right)}{(b-s)}\right] \\
& \times f_{j}\left(y_{1}\left(s+v_{1}-1\right), \ldots, y_{n}\left(s+v_{n}-1\right)\right) \\
= & 0 .
\end{aligned}
$$

Finally, when $0 \leq t_{j}-v_{j}+1 \leq s \leq b+1$,

$$
G_{j}\left(t_{j}, s\right)=\frac{1}{\Gamma\left(v_{j}\right)}\left(v_{j}-1\right)\left(t_{j}-v_{j}+3\right)\left(v_{j}+b-s-1\right) \stackrel{v_{j}-2}{\longrightarrow},
$$

then

$$
\Delta_{t_{j}}^{2} G_{j}\left(t_{j}, s\right)=0
$$

Therefore, we can get

$$
\Delta^{2} T_{j}\left(y_{1}, \ldots, y_{n}\right)\left(v_{j}-3\right)=0 .
$$

So the boundary conditions are satisfied, which completes the proof.

Finally, to accomplish proof of our main results, we state cones theory. In particular, we require the following well-known fixed point theorem for cons in [20].

Theorem 2.9 [20] Let $\mathcal{B}$ a Banach space and let $\mathcal{K} \subseteq \mathcal{B}$ be a cone. Assume that $\Omega_{1}$ and $\Omega_{2}$ are bounded open sets contained in $\mathcal{B}$ such that $0 \in \Omega_{1}$ and $\bar{\Omega}_{1} \subseteq \Omega_{2}$. Assume further that $T: \mathcal{K} \cap\left(\bar{\Omega}_{2} \backslash \Omega_{1}\right) \rightarrow \mathcal{K}$ is a completely continuous operator. If either

(i) $\|T y\| \leq\|y\|$ for $y \in \mathcal{K} \cap \partial \Omega_{1}$ and $\|T y\| \geq\|y\|$ for $y \in \mathcal{K} \cap \partial \Omega_{2}$, or

(ii) $\|T y\| \geq\|y\|$ for $y \in \mathcal{K} \cap \partial \Omega_{1}$ and $\|T y\| \leq\|y\|$ for $y \in \mathcal{K} \cap \partial \Omega_{2}$, then the operator $T$ has at least one fixed point in $\mathcal{K} \cap\left(\bar{\Omega}_{2} \backslash \Omega_{1}\right)$.

\section{Main results}

In this section, we state and prove the existence of at least two positive solutions regarding FBVP (1.1)-(1.2). Then we conclude this section with examples to illustrate our main results. First, denote

$$
\alpha_{j}=\sum_{s=0}^{b+1} G_{j}\left(v_{j}+b, s\right), \quad \beta_{j}=\sum_{s=\left[\frac{v_{j}+b}{4}-v_{j}+1\right]}^{\left[\frac{3\left(v_{j}+b\right)}{4}-v_{j}+1\right]} G_{j}\left(\left[\frac{b-v_{j}}{2}\right]+v_{j}, s\right),
$$




$$
\begin{aligned}
& \Omega_{\xi}=\left\{\left(y_{1}, \ldots, y_{n}\right) \in \Lambda:\left\|\left(y_{1}, \ldots, y_{n}\right)\right\|<\xi\right\}, \\
& \partial \Omega_{\xi}=\left\{\left(y_{1}, \ldots, y_{n}\right) \in \Lambda:\left\|\left(y_{1}, \ldots, y_{n}\right)\right\|=\xi\right\} .
\end{aligned}
$$

For convenience, we now present the conditions that we presume in the sequel.

$\left(\mathrm{H}_{1}\right) \lim _{\left(y_{1}+\cdots+y_{n}\right) \rightarrow 0^{+}} \frac{f_{j}\left(y_{1}, \ldots, y_{n}\right)}{y_{1}+\cdots+y_{n}}=\infty, t_{j} \in\left[v_{j}-2, v_{j}+b\right]_{\mathbb{N}_{v_{j}-2}}, j=1,2, \ldots, n$,

$\left(\mathrm{H}_{2}\right) \lim _{\left(y_{1}+\cdots+y_{n}\right) \rightarrow \infty} \frac{f_{j}\left(y_{1}, \ldots, y_{n}\right)}{y_{1}+\cdots+y_{n}}=\infty, t_{j} \in\left[v_{j}-2, v_{j}+b\right]_{\mathbb{N}_{v_{j}-2}}, j=1,2, \ldots, n$,

$\left(\mathrm{H}_{3}\right) \lim _{\left(y_{1}+\cdots+y_{n}\right) \rightarrow 0^{+}} \frac{f_{j}\left(y_{1}, \ldots, y_{n}\right)}{y_{1}+\cdots+y_{n}}=0, t_{j} \in\left[v_{j}-2, v_{j}+b\right]_{\mathbb{N}_{v_{j}-2}}, j=1,2, \ldots, n$,

$\left(\mathrm{H}_{4}\right) \lim _{\left(y_{1}+\cdots+y_{n}\right) \rightarrow \infty} \frac{f_{j}\left(y_{1}, \ldots, y_{n}\right)}{y_{1}+\cdots+y_{n}}=0, t_{j} \in\left[v_{j}-2, v_{j}+b\right]_{\mathbb{N}_{v_{j}-2}}, j=1,2, \ldots, n$,

$\left(\mathrm{H}_{5}\right) \lim _{\left(y_{1}+\cdots+y_{n}\right) \rightarrow 0^{+}} \frac{f_{j}\left(y_{1}, \ldots, y_{n}\right)}{y_{1}+\cdots+y_{n}}=l_{j}, t_{j} \in\left[v_{j}-2, v_{j}+b\right]_{\mathbb{N}_{v_{j}-2}}, j=1,2, \ldots, n$,

$\left(\mathrm{H}_{6}\right) \lim _{\left(y_{1}+\cdots+y_{n}\right) \rightarrow \infty} \frac{f_{j}\left(y_{1}, \ldots, y_{n}\right)}{y_{1}+\cdots+y_{n}}=L_{j}, t_{j} \in\left[v_{j}-2, v_{j}+b\right]_{\mathbb{N}_{v_{j}-2}}, j=1,2, \ldots, n$,

where $0<l_{j}, L_{j}<+\infty$.

Theorem 3.1 Suppose that there exist two different positive numbers $r_{1}$ and $r_{2}\left(r_{1}<r_{2}\right)$ such that

$$
\begin{aligned}
& \max _{0 \leq y_{1}+\cdots+y_{n} \leq r_{1}} f_{j}\left(y_{1}, \ldots, y_{n}\right) \leq \frac{r_{1}}{n \lambda_{j} \alpha_{j}}, \\
& \min _{\frac{1}{4} r_{2} \leq y_{1}+\cdots+y_{n} \leq r_{2}} f_{j}\left(y_{1}, \ldots, y_{n}\right) \geq \frac{r_{2}}{n \lambda_{j} \beta_{j}} .
\end{aligned}
$$

Then the operator $T$ has a fixed point $\left(\bar{y}_{1}, \ldots, \bar{y}_{n}\right) \in \Lambda$ such that

$$
r_{1} \leq\left\|\left(\bar{y}_{1}, \ldots, \bar{y}_{n}\right)\right\| \leq r_{2}
$$

Proof For any $\left(y_{1}, \ldots, y_{n}\right) \in \Omega_{r_{1}}$ and $\left\|\left(y_{1}, \ldots, y_{n}\right)\right\|=r_{1}$, we have

$$
\begin{aligned}
& \left\|T_{j}\left(y_{1}, \ldots, y_{n}\right)\right\| \\
& \quad=\max _{t_{j} \in\left[v_{j}-2, v_{j}+b\right]_{\mathbb{N}_{j}-2}}\left|\lambda_{j} \sum_{s=0}^{b+1} G_{j}\left(t_{j}, s\right) f_{j}\left(y_{1}\left(s+v_{1}-1\right), \ldots, y_{n}\left(s+v_{n}-1\right)\right)\right| \\
& \quad \leq \lambda_{j} \sum_{s=0}^{b+1} \max _{t_{j} \in\left[v_{j}-2, v_{j}+b\right]_{N_{v_{j}-2}}} G_{j}\left(t_{j}, s\right) f_{j}\left(y_{1}\left(s+v_{1}-1\right), \ldots, y_{n}\left(s+v_{n}-1\right)\right) \\
& \quad \leq \lambda_{j} \sum_{s=0}^{b+1} G_{j}\left(v_{j}+b, s\right) \frac{r_{1}}{n \lambda_{j} \alpha_{j}} \\
& \quad=\frac{r_{1}}{n} \\
& \quad=\frac{1}{n}\left\|\left(y_{1}, \ldots, y_{n}\right)\right\|,
\end{aligned}
$$

$j=1, \ldots, n$. That is,

$$
\begin{aligned}
& \left\|T\left(y_{1}, \ldots, y_{n}\right)\left(t_{1}, \ldots, t_{n}\right)\right\| \\
& \quad=\left\|\left(T_{1}\left(y_{1}, \ldots, y_{n}\right)\left(t_{1}\right), \ldots, T_{n}\left(y_{1}, \ldots, y_{n}\right)\left(t_{n}\right)\right)\right\|
\end{aligned}
$$




$$
\begin{aligned}
& =\left\|T_{1}\left(y_{1}, \ldots, y_{n}\right)\right\|+\cdots+\left\|T_{n}\left(y_{1}, \ldots, y_{n}\right)\right\| \\
& \leq \frac{1}{n}\left\|\left(y_{1}, \ldots, y_{n}\right)\right\|+\cdots+\frac{1}{n}\left\|\left(y_{1}, \ldots, y_{n}\right)\right\| \\
& =\left\|\left(y_{1}, \ldots, y_{n}\right)\right\|
\end{aligned}
$$

for $\left(y_{1}, \ldots, y_{n}\right) \in \partial \Omega_{r_{1}}$.

On the other hand, for any $\left(y_{1}, \ldots, y_{n}\right) \in \Omega_{r_{2}}$ and $\frac{v_{j}+b}{4} \leq t_{j} \leq \frac{3\left(v_{j}+b\right)}{4}$, note that $\left[\frac{b-v_{j}}{2}\right]+v_{j} \in$ $\left[\frac{v_{j}+b}{4}, \frac{3\left(v_{j}+b\right)}{4}\right]$, we have

$$
\begin{aligned}
& \left(T_{j}\left(y_{1}, \ldots, y_{n}\right)\right)\left(\left[\frac{b-v_{j}}{2}\right]+v_{j}\right) \\
& =\lambda_{j} \sum_{s=0}^{b+1} G_{j}\left(\left[\frac{b-v_{j}}{2}\right]+v_{j}, s\right) f_{j}\left(y_{1}\left(s+v_{1}-1\right), \ldots, y_{n}\left(s+v_{n}-1\right)\right) \\
& \left.\geq \lambda_{j} \sum_{s=\left[\frac{v_{j}+b}{4}-v_{j}+b\right)}^{4}-v_{j}+1\right] \\
& \geq \lambda_{j} \sum_{s=\left[\frac{v_{j}+b}{4}-v_{j}+1\right]}^{\left[\frac{3\left(v_{j}+b\right)}{4}-v_{j}+1\right]} G_{j}\left(\left[\frac{b-v_{j}}{2}\right]+v_{j}, s\right) f_{j}\left(y_{1}\left(s+v_{1}-1\right), \ldots, y_{n}\left(s+v_{n}-1\right)\right) \\
& =\frac{r_{2}}{n} .
\end{aligned}
$$

Then

$$
\left\|T_{j}\left(y_{1}, \ldots, y_{n}\right)\right\| \geq \frac{r_{2}}{n}=\frac{1}{n}\left\|\left(y_{1}, \ldots, y_{n}\right)\right\|, \quad j=1, \ldots, n .
$$

That is,

$$
\begin{aligned}
\| & T\left(y_{1}, \ldots, y_{n}\right)\left(t_{1}, \ldots, t_{n}\right) \| \\
& =\left\|\left(T_{1}\left(y_{1}, \ldots, y_{n}\right)\left(t_{1}\right), \ldots, T_{n}\left(y_{1}, \ldots, y_{n}\right)\left(t_{n}\right)\right)\right\| \\
& =\left\|T_{1}\left(y_{1}, \ldots, y_{n}\right)\right\|+\cdots+\left\|T_{n}\left(y_{1}, \ldots, y_{n}\right)\right\| \\
& \geq \frac{1}{n}\left\|\left(y_{1}, \ldots, y_{n}\right)\right\|+\cdots+\frac{1}{n}\left\|\left(y_{1}, \ldots, y_{n}\right)\right\| \\
& =\left\|\left(y_{1}, \ldots, y_{n}\right)\right\|
\end{aligned}
$$

for $\left(y_{1}, \ldots, y_{n}\right) \in \partial \Omega_{r_{2}}$.

By the use of Theorem 2.9, there exists $\left(\bar{y}_{1}, \ldots, \bar{y}_{n}\right) \in \Lambda$ such that $T\left(\bar{y}_{1}, \ldots, \bar{y}_{n}\right)=$ $\left(\bar{y}_{1}, \ldots, \bar{y}_{n}\right)$, the proof is complete.

Theorem 3.2 Suppose that conditions $\left(\mathrm{H}_{1}\right)$ and $\left(\mathrm{H}_{2}\right)$ hold. Then, for every $\lambda_{j} \in\left(0, \lambda_{j}^{*}\right)$, FBVP (1.1)-(1.2) has at least two positive solutions, where

$$
\lambda_{j}^{*}=\frac{1}{n \alpha_{j}} \sup _{r>0} \frac{r}{\max _{0 \leq y_{1}+\cdots+y_{n} \leq r} f_{j}\left(y_{1}, \ldots, y_{n}\right)} .
$$


Proof Define the function

$$
\varphi_{j}(r)=\frac{r}{n \alpha_{j} \max _{0 \leq y_{1}+\cdots+y_{n} \leq r} f_{j}\left(y_{1}, \ldots, y_{n}\right)}, \quad j=1, \ldots, n .
$$

It is easy to know that $\varphi_{j}:(0,+\infty) \rightarrow(0,+\infty)$ is a continuous function. From $\left(\mathrm{H}_{1}\right)$, we see that $\lim _{r \rightarrow 0} \frac{r}{f_{j}(r)}=0$, that is, $\lim _{r \rightarrow 0} \frac{r}{n \alpha_{j} f_{j}(r)}=0$, and

$$
0<\varphi_{j}(r)=\frac{r}{n \alpha_{j} \max _{0 \leq y_{1}+\cdots+y_{n} \leq r} f_{j}\left(y_{1}, \ldots, y_{n}\right)} \leq \frac{r}{n \alpha_{j} f_{j}(r)}
$$

so $\lim _{r \rightarrow 0} \varphi_{j}(r)=0$.

From $\left(\mathrm{H}_{2}\right)$, we see further that $\lim _{r \rightarrow \infty} \varphi_{j}(r)=0$. Then there exists $r_{0}>0$ such that $\varphi_{j}\left(r_{0}\right)=\max _{r>0} \varphi_{j}(r)=\lambda_{j}^{*}, j=1, \ldots, n$. For any $\lambda_{j} \in\left(0, \lambda_{j}^{*}\right)$, by the intermediate value theorem, there exist two points $d_{1} \in\left(0, r_{0}\right), d_{2} \in\left(r_{0}, \infty\right)$ such that $\varphi_{j}\left(d_{1}\right)=\varphi_{j}\left(d_{2}\right)=\lambda_{j}$. Thus, we have

$$
\begin{aligned}
& f_{j}\left(y_{1}, \ldots, y_{n}\right) \leq \frac{d_{1}}{n \lambda_{j} \alpha_{j}}, \quad y_{1}+\cdots+y_{n} \in\left[0, d_{1}\right], \\
& f_{j}\left(y_{1}, \ldots, y_{n}\right) \leq \frac{d_{2}}{n \lambda_{j} \alpha_{j}}, \quad y_{1}+\cdots+y_{n} \in\left[0, d_{2}\right] .
\end{aligned}
$$

On the other hand, since $\left(\mathrm{H}_{1}\right)$ and $\left(\mathrm{H}_{2}\right)$ hold, there exist $e_{1} \in\left(0, d_{1}\right), e_{2} \in\left(d_{2}, \infty\right)$ such that

$$
\frac{f_{j}\left(y_{1}, \ldots, y_{n}\right)}{y_{1}+\cdots+y_{n}} \geq \frac{4}{n \lambda_{j} \beta_{j}}, \quad y_{1}+\cdots+y_{n} \in\left(0, e_{1}\right] \cup\left[\frac{1}{4} e_{2}, \infty\right) .
$$

Thus

$$
\begin{aligned}
& f_{j}\left(y_{1}, \ldots, y_{n}\right) \geq \frac{e_{1}}{n \lambda_{j} \beta_{j}}, \quad y_{1}+\cdots+y_{n} \in\left[\frac{1}{4} e_{1}, e_{1}\right], \\
& f_{j}\left(y_{1}, \ldots, y_{n}\right) \geq \frac{e_{2}}{n \lambda_{j} \beta_{j}}, \quad y_{1}+\cdots+y_{n} \in\left[\frac{1}{4} e_{2}, e_{2}\right] .
\end{aligned}
$$

Application of Theorem 3.1 and Theorem 2.8 leads to two distinct positive solutions of FBVP (1.1)-(1.2) which satisfy

$$
e_{1} \leq\left\|\left(\bar{y}_{1}, \ldots, \bar{y}_{n}\right)\right\| \leq d_{1}, \quad d_{2} \leq\left\|\left(\bar{y}_{1}^{\prime}, \ldots, \bar{y}_{n}^{\prime}\right)\right\| \leq e_{2} .
$$

The proof is complete.

By the proof of Theorem 3.2, we obtain the following.

Corollary 3.3 If one of conditions $\left(\mathrm{H}_{1}\right)$ and $\left(\mathrm{H}_{2}\right)$ is satisfied, then for every $0<\lambda_{j}<\lambda_{j}^{*}$, FBVP (1.1)-(1.2) has at least one positive solution.

Theorem 3.4 Suppose that $\left(\mathrm{H}_{3}\right),\left(\mathrm{H}_{4}\right)$ hold. Then, for any $\lambda_{j} \geq \lambda_{j}^{* *}, F B V P(1.1)-(1.2)$ has at least two positive solutions, where

$$
\lambda_{j}^{* *}=\frac{1}{n \beta_{j}} \inf _{r>0} \frac{r}{\min _{\frac{1}{4} r \leq y_{1}+\cdots+y_{n} \leq r} f_{j}\left(y_{1}, \ldots, y_{n}\right)} .
$$


Proof Define the function

$$
\psi_{j}(r)=\frac{r}{n \beta_{j} \min _{r / 4 \leq y_{1}+\cdots+y_{n} \leq r} f_{j}\left(y_{1}, \ldots, y_{n}\right)}, \quad j=1, \ldots, n .
$$

We know that $\psi_{j}:(0,+\infty) \rightarrow(0,+\infty)$ is a continuous function. For $\lambda_{j}>\lambda_{j}^{* *}$, there exists $0<e_{3}<+\infty$ such that

$$
f_{j}\left(y_{1}, \ldots, y_{n}\right) \geq \frac{e_{3}}{n \lambda_{j} \beta_{j}}, \quad y_{1}+\cdots+y_{n} \in\left[\frac{1}{4} e_{3}, e_{3}\right] .
$$

By condition $\left(\mathrm{H}_{3}\right)$, there exists $0<d_{3}<e_{3}$ such that

$$
f_{j}\left(y_{1}, \ldots, y_{n}\right) \leq \frac{d_{3}}{n \lambda_{j} \alpha_{j}}, \quad y_{1}+\cdots+y_{n} \in\left[0, d_{3}\right] .
$$

From condition $\left(\mathrm{H}_{4}\right)$, there exists $e_{3}<d_{0}<+\infty$ such that

$$
\frac{f_{j}\left(y_{1}, \ldots, y_{n}\right)}{y_{1}+\cdots+y_{n}} \leq \frac{1}{n \lambda_{j} \alpha_{j}}, \quad y_{1}+\cdots+y_{n} \in\left[d_{0},+\infty\right) .
$$

Let $M_{j}=\max _{0 \leq y_{1}+\cdots+y_{n} \leq d_{0}} f_{j}\left(y_{1}, \ldots, y_{n}\right)$. Choose $d_{4}>d_{0}$ such that $d_{4} \geq \lambda_{j} M_{j} \alpha_{j}$. Then

$$
f_{j}\left(y_{1}, \ldots, y_{n}\right) \leq \frac{d_{4}}{n \lambda_{j} \alpha_{j}}, \quad y_{1}+\cdots+y_{n} \in\left[0, d_{4}\right]
$$

By Theorem 3.1 and Theorem 2.8, the proof is complete.

From the proof of Theorem 3.4, we get the following.

Corollary 3.5 Suppose that one of conditions $\left(\mathrm{H}_{3}\right)$ and $\left(\mathrm{H}_{4}\right)$ holds. Then, for every $\lambda_{j}>\lambda_{j}^{* *}$, FBVP (1.1)-(1.2) has at least one positive solution.

Theorem 3.6 Suppose that one of the following cases is satisfied:

(1) $\left(\mathrm{H}_{1}\right),\left(\mathrm{H}_{6}\right)$ hold, and $0<\lambda_{j}<\frac{1}{n \alpha_{j} L_{j}}$;

(2) $\left(\mathrm{H}_{2}\right),\left(\mathrm{H}_{5}\right)$ hold, and $0<\lambda_{j}<\frac{1}{n \alpha_{j} l_{j}}$.

Then FBVP (1.1)-(1.2) has at least one positive solution.

Proof (1) From $\left(\mathrm{H}_{6}\right)$ (namely $\lim _{\left(y_{1}+\cdots+y_{n}\right) \rightarrow \infty} \frac{f_{j}\left(y_{1}, \ldots, y_{n}\right)}{y_{1}+\cdots+y_{n}}=L_{j}, t_{j} \in\left[v_{j}-2, v_{j}+b\right]_{\mathbb{N}_{v_{j}-2}}, j=1,2$, $\ldots, n)$, for any $\epsilon_{j}>0$, there exists a number $R_{0}>0$, for $y_{1}+\cdots+y_{n} \in\left(R_{0},+\infty\right)$, we have

$$
f_{j}\left(y_{1}, \ldots, y_{n}\right)<\left(L_{j}+\epsilon_{j}\right)\left(y_{1}+\cdots+y_{n}\right)
$$

Let $M_{j}=\max _{0 \leq y_{1}+\cdots+y_{n} \leq R_{0}} f_{j}\left(y_{1}, \ldots, y_{n}\right)$. Choose $R>\max \left\{R_{0}, \frac{M_{j}}{L_{j}+\epsilon_{j}}\right\}$, then

$$
f_{j}\left(y_{1}, \ldots, y_{n}\right)<\left(L_{j}+\epsilon_{j}\right)\left(y_{1}+\cdots+y_{n}\right)
$$

for $y_{1}+\cdots+y_{n} \in[0, R]$. As arbitrarily of $\epsilon_{j}$, so $f_{j}\left(y_{1}, \ldots, y_{n}\right) \leq L_{j} R$. Note that $0<\lambda_{j}<\frac{1}{n \alpha_{j} L_{j}}$, then

$$
\lambda_{j}^{*}=\frac{1}{n \alpha_{j}} \sup _{R>0} \frac{R}{\max _{0 \leq y_{1}+\cdots+y_{n} \leq R} f(y)}>\frac{1}{n \alpha_{j}} \frac{R}{L R}=\frac{1}{n \alpha_{j} L_{j}}>\lambda_{j}>0 .
$$


Namely $0<\lambda_{j}<\lambda_{j}^{*}$. By means of Corollary 3.3, FBVP (1.1)-(1.2) has at least one positive solution.

The proof is similar to (2) and hence omitted.

Similarly, we have the following.

Theorem 3.7 Suppose that one of the following cases is satisfied:

(1) $\left(\mathrm{H}_{3}\right),\left(\mathrm{H}_{6}\right)$ hold, and $\frac{4}{n L_{j} \beta_{j}}<\lambda_{j}<+\infty$;

(2) $\left(\mathrm{H}_{4}\right),\left(\mathrm{H}_{5}\right)$ hold, and $\frac{4}{n l_{j} \beta_{j}}<\lambda_{j}<+\infty$.

Then FBVP (1.1)-(1.2) has at least one positive solution.

Theorem 3.8 Suppose that conditions $\left(\mathrm{H}_{5}\right)$ and $\left(\mathrm{H}_{6}\right)$ hold. If $\lambda_{j}$ satisfies $\frac{4}{n \beta_{j} L_{j}}<\lambda_{j}<\frac{1}{n \alpha_{j} l_{j}}$ or $\frac{4}{n \beta_{j} l_{j}}<\lambda_{j}<\frac{1}{n \alpha_{j} L_{j}}$, then FBVP (1.1)-(1.2) has at least one positive solution.

Proof Suppose that $\frac{4}{n \beta_{j} L_{j}}<\lambda_{j}<\frac{1}{n \alpha_{j} l_{j}}$ holds. Choose $\epsilon_{j}>0$ such that $\frac{4}{n \beta_{j}\left(L_{j}-\epsilon_{j}\right)} \leq \lambda_{j} \leq \frac{1}{n \alpha_{j}\left(l_{j}+\epsilon_{j}\right)}$. With condition $\left(\mathrm{H}_{5}\right)$, there exists $\tau>0$ such that $f_{j}\left(y_{1}, \ldots, y_{n}\right) \leq\left(l_{j}+\epsilon_{j}\right)\left(y_{1}+\cdots+y_{n}\right)$ for $y_{1}+\cdots+y_{n} \in(0, \tau)$. Thus, for $y_{1}+\cdots+y_{n} \in \partial \Omega_{\tau}$,

$$
\begin{aligned}
& \left\|T_{j}\left(y_{1}, \ldots, y_{n}\right)\right\| \\
& =\max _{t_{j} \in\left[v_{j}-2, v_{j}+b\right]_{\mathbb{N}_{v_{j}-2}}}\left|\lambda_{j} \sum_{s=0}^{b+1} G_{j}\left(t_{j}, s\right) f_{j}\left(y_{1}\left(s+v_{1}-1\right), \ldots, y_{n}\left(s+v_{n}-1\right)\right)\right| \\
& \quad \leq \lambda_{j}\left(l_{j}+\epsilon_{j}\right)\left(y_{1}+\cdots+y_{n}\right) \sum_{s=0}^{b+1} \max _{t_{j} \in\left[v_{j}-2, v_{j}+b\right]_{\mathbb{N}_{v_{j}-2}}} G_{j}\left(t_{j}, s\right) \\
& \quad=\lambda_{j}\left(l_{j}+\epsilon_{j}\right)\left(y_{1}+\cdots+y_{n}\right) \sum_{s=0}^{b+1} G_{j}\left(v_{j}+b, s\right) \\
& \quad \leq \lambda_{j} \alpha_{j} \frac{\tau}{n \lambda_{j} \alpha_{j}} \\
& =\frac{\tau}{n} \\
& =\frac{1}{n}\left\|\left(y_{1}, \ldots, y_{n}\right)\right\|
\end{aligned}
$$

$j=1, \ldots, n$. That is,

$$
\begin{aligned}
\| & T\left(y_{1}, \ldots, y_{n}\right)\left(t_{1}, \ldots, t_{n}\right) \| \\
& =\left\|\left(T_{1}\left(y_{1}, \ldots, y_{n}\right)\left(t_{1}\right), \ldots, T_{n}\left(y_{1}, \ldots, y_{n}\right)\left(t_{n}\right)\right)\right\| \\
& =\left\|T_{1}\left(y_{1}, \ldots, y_{n}\right)\right\|+\cdots+\left\|T_{n}\left(y_{1}, \ldots, y_{n}\right)\right\| \\
& \leq \frac{1}{n}\left\|\left(y_{1}, \ldots, y_{n}\right)\right\|+\cdots+\frac{1}{n}\left\|\left(y_{1}, \ldots, y_{n}\right)\right\| \\
& =\left\|\left(y_{1}, \ldots, y_{n}\right)\right\|
\end{aligned}
$$

for $\left(y_{1}, \ldots, y_{n}\right) \in \partial \Omega_{\tau}$.

By condition $\left(\mathrm{H}_{6}\right)$, there exists $R_{1}>0$ such that $f_{j}\left(y_{1}, \ldots, y_{n}\right) \geq\left(L_{j}-\epsilon_{j}\right)\left(y_{1}+\cdots+y_{n}\right)$ for $y_{1}+\cdots+y_{n} \geq \frac{1}{4} R_{1}$. 
Let $R_{2}=\max \left\{2 \tau, R_{1}\right\}$, for $\left(y_{1}, \ldots, y_{n}\right) \in \partial \Omega_{R_{2}}$, we get

$$
\begin{aligned}
& \left(T_{j}\left(y_{1}, \ldots, y_{n}\right)\right)\left(\left[\frac{b-v_{j}}{2}\right]+v_{j}\right) \\
& =\lambda_{j} \sum_{s=0}^{b+1} G_{j}\left(\left[\frac{b-v_{j}}{2}\right]+v_{j}, s\right) f_{j}\left(y_{1}\left(s+v_{1}-1\right), \ldots, y_{n}\left(s+v_{n}-1\right)\right) \\
& \geq \lambda_{j}\left(L_{j}-\epsilon_{j}\right) \sum_{s=\left[\frac{v_{j}+b}{4}-v_{j}+1\right]}^{\left[\frac{3\left(v_{j}+b\right)}{4}-v_{j}+1\right]} G_{j}\left(\left[\frac{b-v_{j}}{2}\right]+v_{j}, s\right)\left(y_{1}+\cdots+y_{n}\right) \\
& \left.\geq \lambda_{j} \frac{1}{4}\left(L_{j}-\epsilon_{j}\right) R_{2} \sum_{s=\left[\frac{3\left(v_{j}+b\right)}{4}-v_{j}+1\right]}^{4}-v_{j}+1\right] \\
& \geq \frac{R_{2}}{n} .
\end{aligned}
$$

Then

$$
\left\|T_{j}\left(y_{1}, \ldots, y_{n}\right)\right\| \geq \frac{R_{2}}{n}=\frac{1}{n}\left\|\left(y_{1}, \ldots, y_{n}\right)\right\|, \quad j=1, \ldots, n
$$

That is,

$$
\begin{aligned}
\| & T\left(y_{1}, \ldots, y_{n}\right)\left(t_{1}, \ldots, t_{n}\right) \| \\
& =\left\|\left(T_{1}\left(y_{1}, \ldots, y_{n}\right)\left(t_{1}\right), \ldots, T_{n}\left(y_{1}, \ldots, y_{n}\right)\left(t_{n}\right)\right)\right\| \\
& =\left\|T_{1}\left(y_{1}, \ldots, y_{n}\right)\right\|+\cdots+\left\|T_{n}\left(y_{1}, \ldots, y_{n}\right)\right\| \\
& \geq \frac{1}{n}\left\|\left(y_{1}, \ldots, y_{n}\right)\right\|+\cdots+\frac{1}{n}\left\|\left(y_{1}, \ldots, y_{n}\right)\right\| \\
& =\left\|\left(y_{1}, \ldots, y_{n}\right)\right\|
\end{aligned}
$$

for $\left(y_{1}, \ldots, y_{n}\right) \in \partial \Omega_{R_{2}}$

By using Theorem 2.9, we obtain the conclusion.

A similar proof holds when $\frac{4}{n \beta_{j} l_{j}}<\lambda_{j}<\frac{1}{n \alpha_{j} L_{j}}$. The proof is complete.

We now present an example illustrating the sorts of boundary conditions that can be treated by Theorem 3.2.

Example 3.1 Consider the following boundary value problems:

$$
\left\{\begin{array}{l}
\Delta^{\frac{17}{8}} y_{1}(t)=-\lambda_{1} f_{1}\left(y_{1}\left(t+\frac{9}{8}\right), y_{2}\left(t+\frac{17}{16}\right)\right) \\
\Delta^{\frac{33}{16}} y_{2}(t)=-\lambda_{2} f_{2}\left(y_{1}\left(t+\frac{9}{8}\right), y_{2}\left(t+\frac{17}{16}\right)\right) \\
y_{1}\left(-\frac{7}{8}\right)=\Delta y_{1}\left(\frac{169}{8}\right)=\Delta^{2} y_{1}\left(-\frac{7}{8}\right)=0 \\
y_{2}\left(-\frac{15}{16}\right)=\Delta y_{2}\left(\frac{337}{16}\right)=\Delta^{2} y_{2}\left(-\frac{15}{16}\right)=0
\end{array}\right.
$$

where $b=19, v_{1}=\frac{17}{8}, v_{2}=\frac{33}{16}$, we take

$$
f_{1}\left(y_{1}, y_{2}\right)=\frac{1}{64 \sqrt{2}}\left(y_{1}+y_{2}\right)^{\frac{1}{2}}+\left(y_{1}+y_{2}\right)^{2}, \quad f_{2}\left(y_{1}, y_{2}\right)=\frac{1}{25}\left(y_{1}+y_{2}\right)^{\frac{1}{2}}+\frac{1}{64}\left(y_{1}+y_{2}\right)^{\frac{3}{2}}
$$


$f_{1}, f_{2}:[0,+\infty) \times[0,+\infty) \rightarrow[0,+\infty)$, and $y_{1}$ is defined on the time scale $\left\{-\frac{7}{8}, \frac{1}{8}, \ldots, \frac{169}{8}\right\}, y_{2}$ is defined on the time scale $\left\{-\frac{15}{16}, \frac{1}{16}, \ldots, \frac{337}{16}\right\} . f_{1}$ and $f_{2}$ satisfy conditions of Theorem 3.2 . A computation shows that $\lambda_{1}^{*} \approx 0.01456, \lambda_{2}^{*} \approx 0.032845$, then, for every $\lambda_{j} \in\left(0, \lambda_{j}^{*}\right)(j=$ $1,2)$, problem (3.1) has at least two positive solutions.

\section{Competing interests}

The authors declare that they have no competing interests.

\section{Authors' contributions}

SK conceived of the study and participated in its design. HC drafted the manuscript and participated in its design and coordination. JG participated in the sequence correction. All authors read and approved the final manuscript.

\section{Acknowledgements}

The authors are very grateful to the reviewers for their valuable suggestions and useful comments, which led to an improvement of this paper. Project was supported by the National Natural Science Foundation of China (Grant No. 11271235) and Shanxi Datong University Institute (2009-Y-15, 2010-B-01, 2013K5) and the Development Foundation of Higher Education Department of Shanxi Province $(20101109,20111020)$

Received: 4 November 2014 Accepted: 10 April 2015 Published online: 06 May 2015

\section{References}

1. Abdeljawad, T: On Riemann and Caputo fractional differences. Appl. Comput. Math. 62, 1602-1611 (2011)

2. Holm, M: Sum and difference compositions in discrete fractional calculus. CUBO 13(3), 153-184 (2011)

3. Atici, FM, Sengül, S: Modeling with fractional difference equations. J. Math. Anal. Appl. 369, 1-9 (2010)

4. Abdeljawad, T, Baleanu, D: Fractional differences and integration by parts. J. Comput. Anal. Appl. 13(3), 574-582 (2011)

5. Jarad, F, Abdeljawad, T, Baleanu, D, Biçen, K: On the stability of some discrete fractional nonautonomous systems Abstr. Appl. Anal. 2012, Article ID 476581 (2012)

6. Mohammadi, H, Rezapour, S: Existence results for nonlinear fractional differential equations on ordered gauge spaces. J. Adv. Math. Stud. 6(2), 154-158 (2013)

7. Wu, GC, Baleanu, D: Discrete fractional logistic map and its chaos. Nonlinear Dyn. 75, 283-287 (2014)

8. Wu, GC, Baleanu, D: Chaos synchronization of the discrete fractional logistic map. Signal Process. 102, 96-99 (2014)

9. Chen, HQ, Cui, YQ, Zhao, XL: Multiple solutions to fractional difference boundary value problems. Abstr. Appl. Anal. 2014, Article ID 879380 (2014)

10. Chen, HQ, Jin, Z, Kang, SG: Existence of positive solutions for Caputo fractional difference equation. Adv. Differ. Equ. 2015, 44 (2015)

11. Goodrich, CS: On a fractional boundary value problem with fractional boundary conditions. Appl. Math. Lett. 25, 1101-1105 (2012)

12. Goodrich, CS: On discrete sequential fractional boundary value problems. J. Math. Anal. Appl. 385, 111-124 (2012)

13. Goodrich, CS: On a discrete fractional three-point boundary value problem. J. Differ. Equ. Appl. 18, $397-415$ (2012)

14. Atici, FM, Eloe, PW: Linear systems of fractional nabla difference equations. Rocky Mt. J. Math. 41, 353-370 (2011)

15. Baleanu, D, Rezapour, S, Salehi, S: A k-dimensional system of fractional finite difference equations. Abstr. Appl. Anal. 2014, Article ID 312578 (2014)

16. Goodrich, CS: Existence of a positive solution to a system of discrete fractional boundary value problems. Appl. Comput. Math. 217, 4740-4753 (2011)

17. Goodrich, CS: Nonlocal systems of BVPs with asymptotically superlinear boundary conditions. Comment. Math. Univ. Carol. 53, 79-97 (2012)

18. Dahal, R, Duncan, D, Goodrich, CS: Systems of semipositone discrete fractional boundary value problems. J. Differ Equ. Appl. (2013). doi:10.1080/10236198.2013.856073

19. Kang, SG, Zhao, XH, Chen, HQ: Positive solutions for boundary value problems of fractional difference equations depending on parameters. Adv. Differ. Equ. 2013, 376 (2013)

20. Agarwal, R, Meehan, M, O'Regan, D: Fixed Point Theory and Applications. Cambridge University Press, Cambridge (2001) 